\title{
Development of Personalized Fitting Device With 3-Dimensional Solution for Prevention of NIV Oronasal Mask-Related Pressure Ulcers
}

\author{
Maiko Shikama MHS RN, Gojiro Nakagami PhD RN, Hiroshi Noguchi PhD, \\ Taketoshi Mori PhD, and Hiromi Sanada PhD RN WOCN
}

\begin{abstract}
BACKGROUND: Pressure ulcers related to oronasal masks used with noninvasive ventilation (NIV), along with patient discomfort, occur due to improper fit of the mask. We developed a personalized fitting device using a 3-dimensional (3D) scanning solution to prevent the formation of NIV mask-related pressure ulcers. This study aimed to evaluate the effectiveness of the proposed personalized fitting device. METHODS: We conducted a randomized crossover experimental study of 20 healthy participants to study the use of this personalized fitting device between the face and an NIV mask designed with 3D solutions. The fitting device was not used under the NIV mask for the control. The outcome measures were the presence of blanchable erythema, standardized redness intensity, discomfort level, and contact pressure. RESULTS: The incidence of blanchable erythema and standardized redness intensity values were significantly lower for subjects who used the fitting device when worn for $30 \mathrm{~min}(P<.001)$. The discomfort levels at the forehead, nasal bridge, and both cheeks, as well as leakage, were significantly reduced as well $(P=.008, P<.001, P=.001$, $P=.002, P=.001, P=.02, P<.001, P<.001, P<.001)$. Contact pressure at the nasal bridge, where pressure ulcers most frequently develop, was significantly decreased with the fitting device $(P<.001)$. CONCLUSIONS: Personalized fitting devices that incorporate 3D scanning solutions may contribute to the prevention of NIV mask-related pressure ulcers and the reduction of discomfort. Key words: adverse effects; critical care; imaging, three-dimensional; intensive care units; mask fit; medical device-related pressure ulcers; noninvasive ventilation; pressure ulcer; printing, threedimensional. [Respir Care 2018;63(8):1024-1032. (C) 2018 Daedalus Enterprises]
\end{abstract}

\section{Introduction}

Medical device-related pressure ulcers cause pain, loss of function, and infection, resulting in extended hospital stays and increased costs. ${ }^{1}$ Medical device-related pressure ulcers are recognized as adverse events in health care,

\footnotetext{
Ms Shikama and Drs Nakagami and Sanada are affiliated with the Department of Gerontological Nursing/Wound Care Management, The University of Tokyo, Japan. Drs Nakagami, Noguchi, Mori, and Sanada are affiliated with the Global Nursing Research Center, The University of Tokyo, Japan. Drs Noguchi and Mori are also affiliated with the Department of Life Support Technology (Molten), The University of Tokyo, Tokyo, Japan.
}

This study was supported by a grant from Japanese Society of Wound, Ostomy and Continence Management and the JSPS KAKENHI (grant no. $15 \mathrm{~K} 15784)$. The authors have disclosed no other conflicts of interest. but they should be a never event because such pressure ulcers are largely preventable. ${ }^{2,3}$ In the United States, the development of medical device-related pressure ulcers can lead to significant economic costs. ${ }^{3}$

Despite the application of best practices to prevent medical device-related pressure ulcers, their prevalence among in-patients is as high as $2.0-6.1 \%$ in the ICU setting, and the proportion of medical device-related pressure ulcers to total hospital-acquired pressure ulcers is $20.0-47.1 \% .^{4,5}$ Therefore, more effective strategies to prevent pressure

\footnotetext{
Correspondence: Hiromi Sanada PhD RN WOCN, Faculty of Medicine Building 5-307, 7-3-1, Hongo, Bunkyo-ku, Tokyo 113-0033. E-mail: hsanada-tky@umin.ac.jp.
}

DOI: 10.4187/respcare.05691 
ulcers related to the use of medical devices are urgently needed.

Among these injuries, wounds caused by the improper fit an oronasal mask for noninvasive ventilation (NIV) can drastically reduce the patient's quality of life and sometimes become life-threatening. ${ }^{6-9}$ The improper fit of the NIV mask also causes patient discomfort. ${ }^{7}$ An improper fit can lead to pressure ulcers, but sometimes even a properly fitted mask can cause these issues. Blanchable erythema, which signals the potential for pressure ulcer development, can occur quickly, even with short-term use of an improperly fitted NIV mask, especially on the nasal bridge where NIV mask-related pressure ulcers most frequently develop because of thin, delicate, and sensitive skin. ${ }^{6,10-12}$

To achieve a proper fit, a dressing can be used as a fitting material to eliminate the gap between the patient's face and the mask to redistribute the pressure as well as to minimize leakage volume. ${ }^{9,13,14}$ In addition, clinicians are educated on the proper application of the NIV mask, especially correct mask placement and angle. ${ }^{13,14}$ Nevertheless, previous studies have reported that the cumulative incidence of NIV mask-related pressure ulcers is as high as $20.0-60.8 \%$, which is mostly due to difficulty in appropriately fitting the mask. ${ }^{6,11}$

Raurell-Torreda et al ${ }^{15}$ associated the occurrence of NIV mask-related pressure ulcers with excessive air leaks. They also reported that clinicians tend to press the NIV mask tightly against the patient's face to decrease air leaks. Experimental studies have determined that a minimum contact pressure to prevent excessive air leaks was 1-2 $\mathrm{cm} \mathrm{H}_{2} \mathrm{O}$ above inspiratory pressure. ${ }^{16,17}$ However, clinicians do not have a way to confirm the degree of contact pressure required when fitting an NIV mask in the clinical setting.

Thus, appropriate training is needed to properly apply a mask to various face shapes, while minimizing leakage volume and avoiding over-tightening the straps. ${ }^{13,14,18,19} \mathrm{In}$ particular, it may be difficult to fit the mask on emaciated patients, patients using a nasogastric tube, or those who are unable to insert dentures, an issue that can increase the risk of leakage volume. ${ }^{13}$ Therefore, personalization of the fitting material to fill the gap between the face and mask is necessary to achieve a proper fit.

Objective measurements of the face and mask are required to calculate the gap to design a personalized fitting device for each patient. For this purpose, computed tomography, magnetic resonance imaging, or a 3-dimensional (3D) scanner can be used. ${ }^{20}$ The use of a 3D scanner was the focus of this study because such a scanner can be applied broadly to patients, from the elderly to young children, because there is no risk of radiation exposure and no need for sedation of the critically ill. Furthermore, the required shape can be easily and immediately obtained with the use of a 3D scanner. ${ }^{10,21,22}$

\section{QUICK LOOK}

\section{Current knowledge}

Noninvasive oronasal mask-related pressure ulcers can drastically reduce the patient's quality of life and sometimes become life threatening due to improper fitting of the mask. Despite training for mask fitting, mask-related pressure ulcers still occur.

\section{What this paper contributes to our knowledge}

The use of the proposed personalized fitting device with the aid of 3D scanning solutions reduced the incidence of blanchable erythema, standardized redness intensity, discomfort level, and contact pressure on the nasal bridge in healthy participants. A personalized fitting device is one of the options to achieve the proper fit of the mask to prevent mask-related pressure ulcers.

There have been some attempts to produce personalized masks or mask cushions with 3D technology to improve patient discomfort due to an NIV nasal mask. ${ }^{23,24}$ Generally, the use of an NIV nasal mask is preferable for patients with obstructive sleep apnea who need long-term ventilation. ${ }^{25}$ On the other hand, an NIV oronasal mask is preferred for critically ill patients. ${ }^{26}$ Manufacturing a personalized NIV oronasal mask is difficult because the facial shapes around both the nose and mouth need to be considered simultaneously. Although such patients are at a high risk for mask-related pressure ulcers, no study has reported the production of a personalized fitting device. ${ }^{27-29}$

In this study, a personalized fitting device is proposed to fill the gap between the face and the mask to achieve a proper fit using 3D solutions. The objective of this randomized crossover experimental study was to determine whether a personalized fitting device could reduce the incidence of blanchable erythema and discomfort level, as well as redistribute the contact pressure.

\section{Methods}

\section{Workflow for Producing a Personalized Fitting Device}

To ensure that the shape of the fitting device was identical to the gap between the face and mask, both surfaces were scanned. To calculate the gap between the face and mask, the mask was fit to the face using 3D software (Artec Studio 10, Artec3D, Luxembourg). Then a mold was produced with a 3D printer (Replicator 2X; MakerBot Industries, Brooklyn, New York). Finally, silicone was cast into the mold to create a personalized fitting device (Fig. 1). 


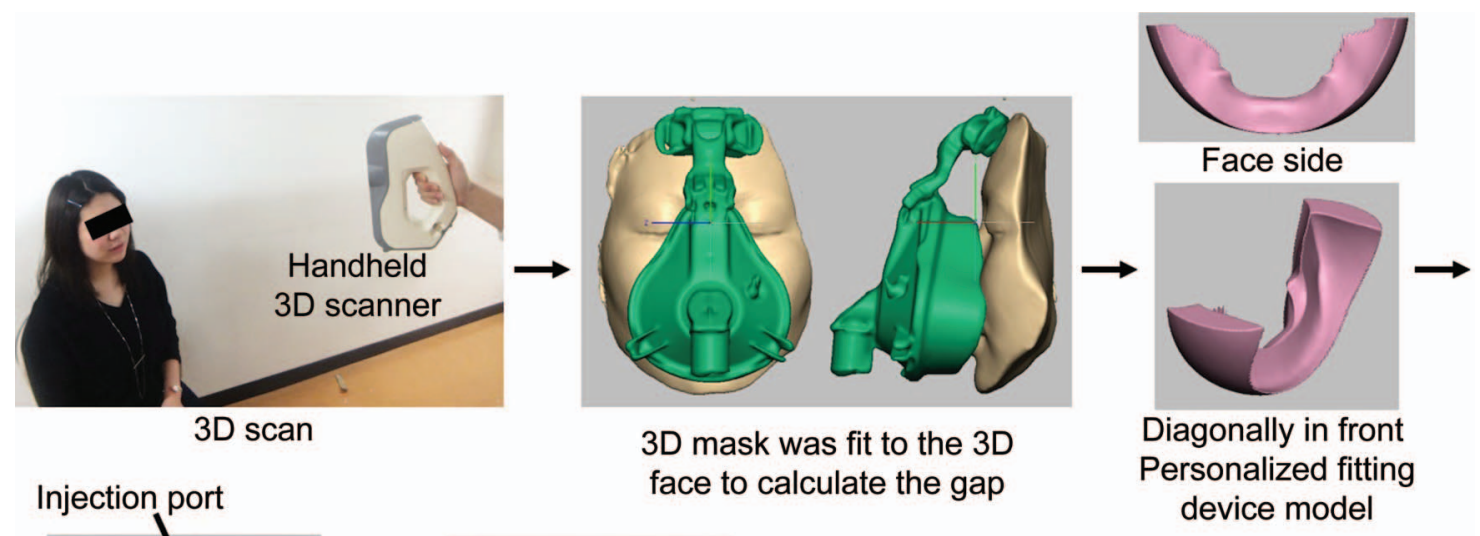

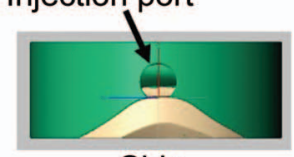

Side

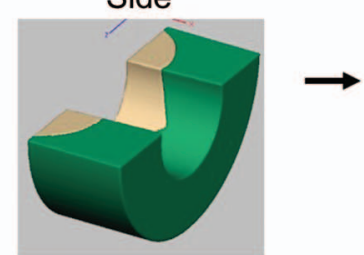

Diagonally in front Mold model

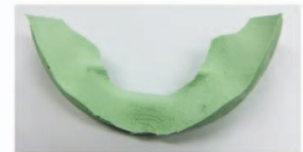

Face side

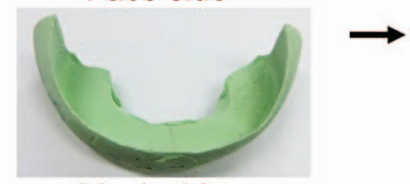

Mask side

Personalized fitting device

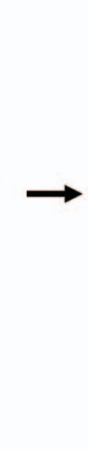

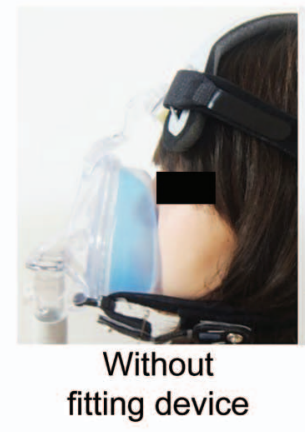

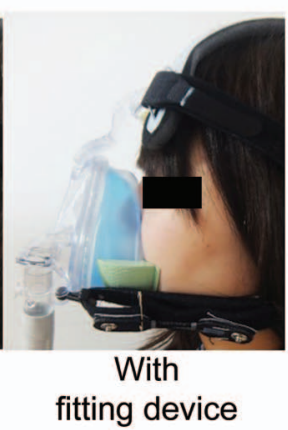

Fig. 1. Overall workflow of producing a personalized fitting device.

\section{Acquisition of Face and Mask Data}

The shapes of the face and NIV oronasal mask (AF811 Oro-Nasal Mask (medium), Philips Respironics, Murrysville, Pennsylvania) were scanned separately using an Eva 3D Scanner (Artec3D). The accuracy and resolution of the scanner were $0.1 \mathrm{~mm}$ and $0.5 \mathrm{~mm}$, respectively. When scanning the face, the participant was seated on a chair with the mouth open, as though wearing an NIV mask. The mask was painted using a surfacer (Tamiya, Shizuoka, Japan) to more easily scan the mask.

The fitting device design was based on a clinically ideal fitting so that the mask would float on the face with minimum pressure and leakage volume. A previous study suggested that the positive distance (overlap) between the 3D face and mask may cause NIV maskrelated pressure ulcers. ${ }^{12}$ In light of these findings, pilot studies to achieve ideal mask fitting were performed to determine the best position between the face and mask using 3D software. The negative distance between the nasal bridge and the mask (ie, the space with no contact) was the most important to address to prevent these pressure ulcers. Additionally, to maintain the shape of the fitting device, the device itself should be made of a material harder than that of the NIV mask. However, as the nose is most susceptible to NIV mask-related pres- sure ulcers, ${ }^{6,11}$ we focused on the gap between the chin and the mask when creating the fitting device so that it did not come into contact with the area surrounding the nose (Fig. 2). Using 3D software, the 3D face and mask scans were aligned so that the mask floated on facial sites other than the chin.

\section{Personalized Fitting Device and Mold}

These 3D data were transformed into solid models using Geomagic Sculpt 3D software (3D Systems, Rock Hill, South Carolina). Torus clay was placed between the face and mask models, then the face and mask models were subtracted from the torus clay using a Boolean operation and unnecessary parts were removed. A mold was designed according to 2 requirements: de-modeling and casting. The personalized fitting device model was subtracted from the mold block. The mold model was divided into top and bottom sections with an injection port created in the top section. A 3D printer (MakerBot Industries) was used to produce the molds. Complete production of these pieces took $\sim 5 \mathrm{~h}$. Each personalized fitting device was created by casting a silicone (Duplicone; SHOFU, Kyoto, Japan) into the mold. The silicone was composed of 2 types that cured at room temperature in $30 \mathrm{~min}$. 
A

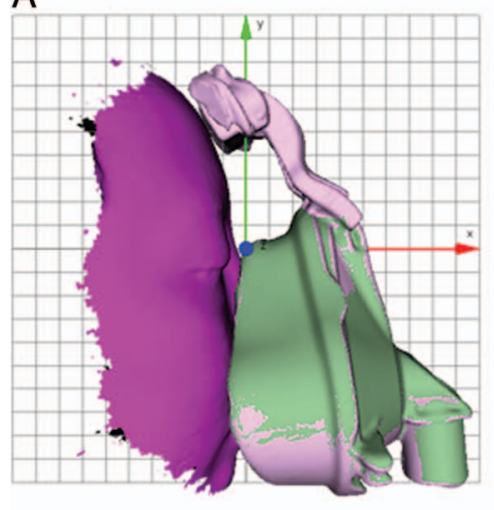

B

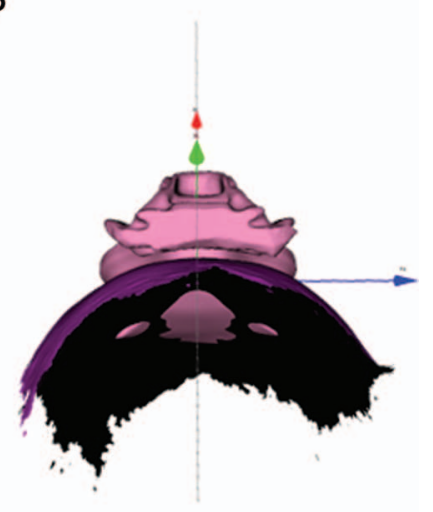

C

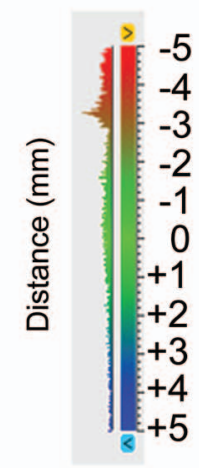

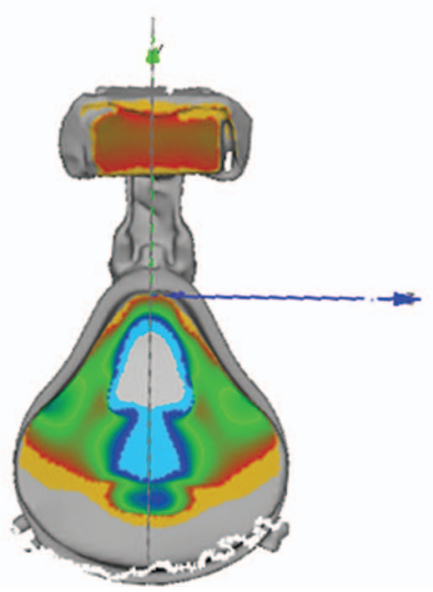

Fig. 2. An example of the positioning of the noninvasive ventilation mask on a face model, as shown using 3D software. (A) Side view of the positioning. (B) Upper view of the positioning. (C) The distance from the face to the mask: a positive distance indicates that the face overlaps the mask, whereas a negative distance indicates that the face does not make contact with the mask.

\section{Experimental Methods}

\section{Study Design and Participants}

A randomized crossover experimental study was conducted in a laboratory at The University of Tokyo. The study protocol was approved by the Ethical Committee of the Graduate School of Medicine, The University of Tokyo (Approval No. 11266). The study participants included 27 healthy individuals age $\geq 20$ y recruited between September and November 2016. The exclusion criteria were current infectious respiratory disease, facial skin injury, contact dermatitis in response to medical adhesive tape, photosensitivity, silicone allergy, or intolerance to NIV. After scanning, if the length from the nasal bridge to the inferior border of the lower lip was $>90 \mathrm{~mm}$ or the length from the nasal bridge to the tip of the chin was $<100 \mathrm{~mm}$, the participants were excluded because the mask was too big or small (the large and small sizes should be considered for such subjects). We used only the medium-sized NIV mask to focus on the proof-of-concept in this study. In addition, if the gap between the face and mask was too small to create a personalized fitting device, the participant was excluded because a fitting device was not needed.

\section{Study Protocol (Fig. 3)}

In a randomized, cross-over experimental study, participants with an NIV mask were randomly assigned to 2 groups. Those in the first group initially used a personalized fitting device between the face and the mask and removed it later, whereas the second group used a personalized fitting device later and not initially. The study was divided into 2 phases: one phase involved the evaluation of the presence of blanchable erythema and associated discomfort, whereas the other phase involved the evaluation of contact pressure. Evaluation of the presence of blanchable erythema and discomfort level with or without the fitting device was performed to leave at least 1 day to avoid any carryover effect. In addition, if both evaluations had to be performed on the same day, contact pressure was evaluated after evaluating erythema and discomfort, considering the effects of the application of sensors to their faces. 


\section{Personalized Fitting Device to Prevent Pressure Ulcers}

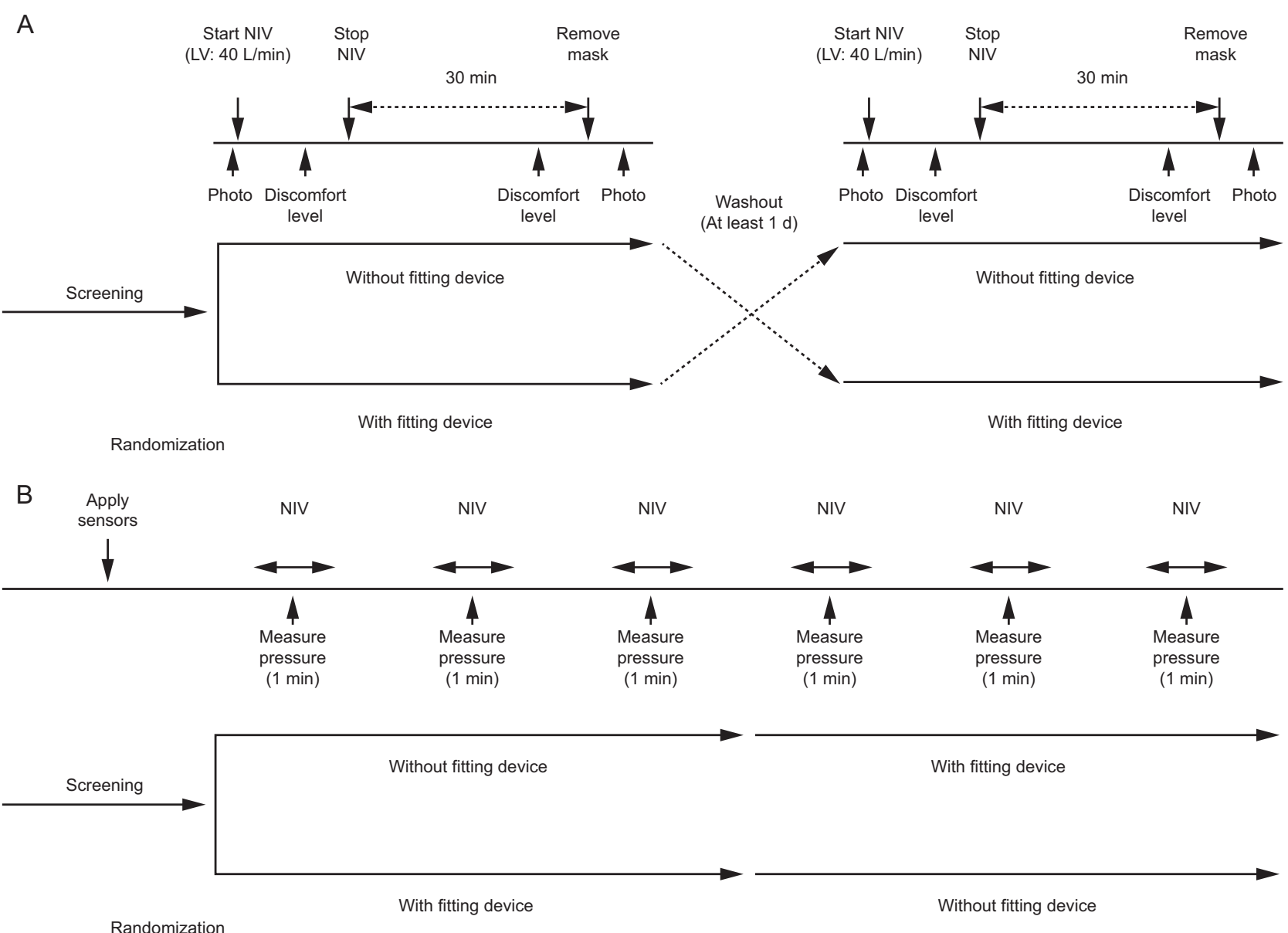

Fig. 3. Experimental protocol. (A) Evaluation of discomfort level and presence of blanchable erythema. (B) Evaluation of contact pressure during NIV at $50 \mathrm{~L} / \mathrm{min}$. NIV = noninvasive ventilation; LV = leak volume.

An A40 bi-level ventilator and an AF811 oronasal mask (Philips Respironics) were used in this study. The inspiratory positive airway pressure was set at $8 \mathrm{~cm} \mathrm{H}_{2} \mathrm{O}$, and the expiratory positive airway pressure was set at $4 \mathrm{~cm} \mathrm{H}_{2} \mathrm{O}$, which were the maximum values considered tolerable to healthy participants. The participants were placed in the supine position on a bed with a head-up angle of $60^{\circ}$ in all experiments. The strap tension was measured and recorded using a Stretchable Strain Sensor (Yamaha, Iwata, Japan) attached on the strap.

\section{Blanchable Erythema and Discomfort Level}

Determining the presence of blanchable erythema, which predisposes the skin to category I pressure ulcers, was adopted as the main study outcome. Moreover, the discomfort level was subjectively evaluated to confirm whether the fitting device reduced discomfort, pain, oppressive feeling, and leakage at the contact site of the mask.
Before applying the NIV mask, each subject's face was photographed. After fitting the mask, the strap tension was adjusted to ensure that the leakage volume was $\sim 40 \mathrm{~L} / \mathrm{min}$, with or without the fitting device. Afterward, the discomfort levels at the nasal bridge, cheeks, chin, and forehead, as well as discomfort caused by leakage, was measured using a 10-point rating scale in which the higher values indicate greater discomfort. After cessation of ventilation, each participant wore the NIV mask for an additional $30 \mathrm{~min}$, with or without the fitting device, because it was difficult for the participants to tolerate long-term ventilation. After $30 \mathrm{~min}$, the discomfort level was evaluated again, except for discomfort caused by leakage, and photographs were acquired a second time.

The presence of blanchable erythema was evaluated by 2 of the researchers, who were blinded to group allocation. Furthermore, the intensity of redness was evaluated by calculating the maximum Erythema Index between with and without the fitting device around the nasal bridge using ImageJ software (National Institutes of Health, 


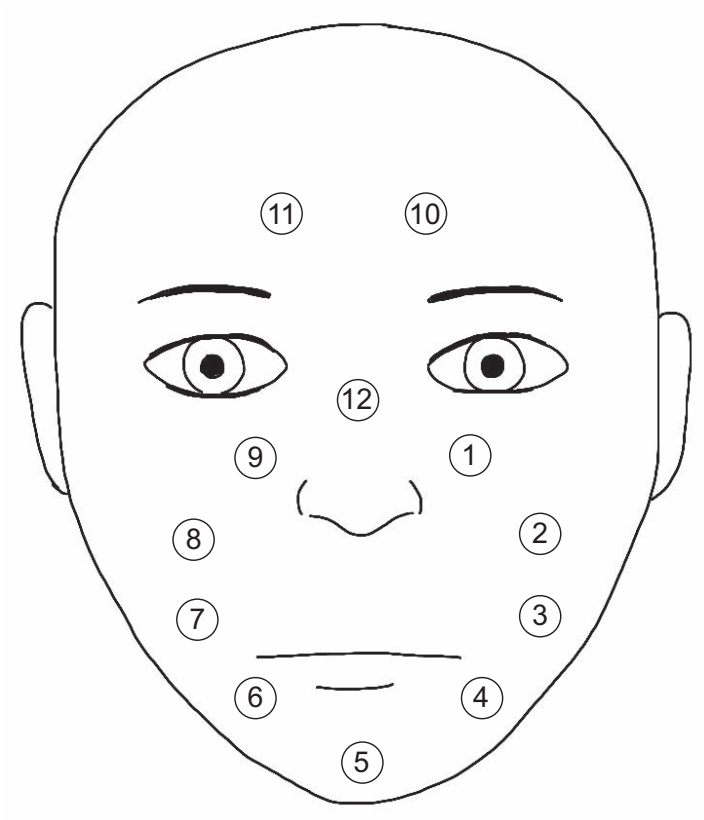

Fig. 4. Sensor location between the mask and face.

Bethesda MD), as described by Yamamoto et al..$^{30}$ The redness intensity around the nasal bridge was divided by that around the apex of the nose for standardization because individual complexions differed depending on the time of day. The apex of the nose was selected as the point of standardization because, while the nose was covered by the NIV mask, no pressure was applied to the nose by the mask.

\section{Contact Pressure}

We hypothesized that contact pressure at the nasal bridge can be reduced and that the contact pressure values on the face can be distributed evenly with use of the fitting device. To test this hypothesis, 12 sensors (Flexi Force; NITTA Corporation, Tokyo, Japan) were attached to the subjects' faces where the mask or fitting device made contact using double-sided adhesive medical tape (Fig. 4). These sensors were thin enough $(0.2 \mathrm{~mm})$ to enable noninvasive measurement. The reliability of contact pressure measurements between the face and mask was previously evaluated. ${ }^{31}$ The coefficient of variation, measured 3 times in this study, was $13.0 \%$ with the fitting device and $12.8 \%$ without. After fitting the NIV mask and adjusting the leakage volume to $\sim 50 \mathrm{~L} / \mathrm{min}$, the contact pressure with or without a fitting device was measured 3 times for $1 \mathrm{~min}$ each. The leakage volume was increased in consideration of the tendency of the sensors to increase the leakage volume.

The distribution of contact pressure was also evaluated to confirm whether the fitting device could efficiently re-
Table 1. Baseline Characteristics of Subjects

\begin{tabular}{lc}
\hline \hline Age, $\mathrm{y}$ & $34.0(29.8-39.0)$ \\
Female, $n(\%)$ & $17(85)$ \\
Body mass index, $\mathrm{kg} / \mathrm{m}^{2}$ & $19.7(19.1-23.3)$ \\
Nasofrontal angle, degree $\left(^{\circ}\right)$ & $144.0(137.8-147.2)$ \\
Length from the nasal root to & $78.4(76.0-81.3)$ \\
$\quad$ the lower lip, mm & \\
\hline$N=$ 20. Data are reported as median (interquartile range). \\
\hline
\end{tabular}

distribute the pressure. The distribution of proportion among the 12 sensors was represented as the coefficient of variation of the proportion of contact pressure for each sensor to the total contact pressure, where a smaller value indicates a more even redistribution of contact pressure on the face.

\section{Statistical Analysis}

Results were summarized as proportions (\%) or medians with interquartile range (IQR). The McNemar test was used to compare the incidence of blanchable erythema with or without use of the fitting device. Continuous variables were compared with or without the fitting device using the Wilcoxon signed-rank test. A $P$ value $<.05$ was considered statistically significant. All statistical analyses were conducted using SPSS, version 20.0 (IBM, Armonk, New York).

\section{Results}

In total, 27 participants were scanned, of whom 7 were excluded because of mismatch in mask size (6 participants) or the gap between the face and mask was too small to create a fitting device (1 participant). Of the 20 participants, $17(85 \%)$ were female, the median age was 34.0 (IQR 29.8-39.0) y, and the median body mass index was 19.7 (IQR 18.9-23.3) kg/m² (Table 1). None of the study participants dropped out or were lost to follow-up.

All participants displayed blanchable erythema when wearing the mask without a fitting device. On the other hand, 15 of 20 subjects (75\%) exhibited no blanchable erythema while wearing a fitting device $(P<.001)$ (Table 2 ). Standardized redness intensity values for subjects with the fitting device were significantly lower than those for subjects without it (median, 1.0 [IQR 0.8-1.1] vs 1.2 [IQR 1.1-1.3], respectively, $P<.001)$.

The discomfort levels were significantly reduced with the use of the fitting device at all evaluated sites except for the chin, both immediately after mask fitting and $30 \mathrm{~min}$ afterward. In addition, the discomfort level of leakage was 


\section{Personalized Fitting Device to Prevent Pressure Ulcers}

Table 2. Presence or Absence of Blanchable Erythema After 30 Min Application of NIV Mask With or Without the Fitting Device on Each Site

\begin{tabular}{lcccr}
\hline \hline & $\begin{array}{c}\text { Present } \\
\text { on Forehead }\end{array}$ & $\begin{array}{c}\text { Present on } \\
\text { Nasal Bridge Only }\end{array}$ & $\begin{array}{c}\text { Present on Nasal } \\
\text { Bridge and Cheeks }\end{array}$ & $\begin{array}{c}\text { Present on Nasal } \\
\text { Bridge and Chin }\end{array}$ \\
\hline $\begin{array}{l}\text { Without fitting device } \\
\text { With fitting device }\end{array}$ & 0 & 16 & 4 & 0 \\
Absence \\
$N=20$
\end{tabular}

Table 3. Discomfort Level

\begin{tabular}{|c|c|c|c|}
\hline & \multicolumn{2}{|c|}{$\begin{array}{l}\text { Numerical } \\
\text { Rating Scale }\end{array}$} & \multirow{2}{*}{$P$} \\
\hline & $\begin{array}{c}\text { Without } \\
\text { Fitting Device }\end{array}$ & $\begin{array}{c}\text { With } \\
\text { Fitting Device }\end{array}$ & \\
\hline \multicolumn{4}{|c|}{ Immediately after } \\
\hline Forehead & $1.5(1.0-3.0)$ & $1.0(0.0-2.0)$ & .008 \\
\hline Nasal bridge & $3.5(3.0-5.3)$ & $2.0(1.0-3.0)$ & $<.001$ \\
\hline Left cheek & $2.0(2.0-4.0)$ & $1.0(0.0-3.0)$ & .001 \\
\hline Right cheek & $2.0(1.0-4.0)$ & $1.0(0.0-2.3)$ & .002 \\
\hline Chin & $2.0(0.8-3.0)$ & $1.5(1.0-2.0)$ & .32 \\
\hline Leakage & $4.5(2.0-6.0)$ & $2.0(1.0-3.0)$ & .001 \\
\hline \multicolumn{4}{|l|}{ After $30 \mathrm{~min}$} \\
\hline Forehead & $2.0(1.0-3.3)$ & $1.0(0.0-2.0)$ & .02 \\
\hline Nasal bridge & $5.5(3.0-6.3)$ & $2.5(1.0-4.0)$ & $<.001$ \\
\hline Left cheek & $3.0(2.0-4.3)$ & $1.5(0.8-2.3)$ & $<.001$ \\
\hline Right cheek & $3.0(2.0-4.0)$ & $1.0(0.0-2.0)$ & $<.001$ \\
\hline Chin & $3.0(1.8-4.0)$ & $1.0(1.0-3.0)$ & .07 \\
\hline
\end{tabular}

significantly reduced by the use of the fitting device immediately after mask fitting $(P=.001)$ (Table 3).

The contact pressure on the nasal bridge was significantly decreased with the use of the fitting device $(P<.001)$. In addition, the contact pressure around the chin was significantly increased $(P=.03$ for Sensor $4, P<.001$ for Sensor 5, and $P<.001$ for Sensor 6). The distribution of contact pressure was significantly lower with the fitting device than without it $(P=.01)$ (Table 4).

The strap tension was significantly higher with the fitting device than without when the leakage volume was maintained at $\sim 50 \mathrm{~L} / \mathrm{min}$ (median $105.0 \mathrm{gf}$ [IQR 58.9-138.1] versus 9.4 gf [IQR 0.0-37.9], respectively, $P<.001$ ).

\section{Discussion}

This is the first study to propose a personalized fitting device created with the aid of 3D solutions to prevent NIV oronasal mask-related pressure ulcers. The incidence of blanchable erythema, standardized redness intensity, dis- comfort level, and contact pressure on the nasal bridge were reduced with the personalized fitting devices, which were created based on accurate measurements by 3D scanning. Therefore, use of this technology is highly expected to prevent medical device-related pressure ulcers if applied clinically for patients with NIV. Furthermore, this study demonstrated an effective use of 3D scanning and printing technology in health care. Because the major cause of medical device-related pressure ulcers is imperfect size or shape of medical devices, ${ }^{29,32}$ providing customized care with the use of 3D scanning solutions in the clinical setting could prevent many instances of medical device-related pressure ulcers.

Although the strap tension with a fitting device was significantly higher than that without it, the contact pressure in most participants did not exceed $40 \mathrm{~mm} \mathrm{Hg}$, which is the threshold of pressure ulcer formation. ${ }^{33}$ Dellweg et $\mathrm{a}^{17}$ found that an increased area of contact between the face and the mask reduced the contact pressure during expiration. Personalized fitting devices can increase the contact area by precisely filling the gap between the face and the NIV mask. Notably, the fitting devices dramatically reduced the contact pressure on the nasal bridge. This can be explained by the even distribution of contact pressure, which was confirmed by the distribution of contact pressure. Therefore, the use of the fitting device may reduce the incidence of mask-related pressure ulcers.

Furthermore, the application of a fitting device might be more effective than a dressing in preventing pressure ulcers. A previous study ${ }^{14}$ evaluated the effectiveness of various dressings for the prevention of NIV mask-related pressure ulcers by checking the skin condition of ICU subjects every $30 \mathrm{~min}$. In the control group, which received no dressing application, 96.7\% developed medical device-related pressure ulcers, while $53.3 \%$ and $40.0 \%$ of subjects who received film or hydrocolloid dressing, respectively, developed medical device-related pressure ulcers. ${ }^{14}$ As indicated by our results, the use of this fitting device considerably reduced the incidence of blanchable erythema, implying potential effectiveness to prevent medical device-related pressure ulcers. Although the incidence of such pressure ulcers was not evaluated, we believe that the use of the proposed fitting device could reduce the 


\section{Personalized Fitting Device to Prevent Pressure Ulcers}

Table 4. Mean Values and Distribution of Contact Pressure

\begin{tabular}{|c|c|c|c|}
\hline Contact Pressure, $\mathrm{mm} \mathrm{Hg}$ & Without Fitting Device & With Fitting Device & $P$ \\
\hline Sensor 1 (upper left side of cheek) & $13.2(11.7-17.7)$ & $13.1(12.1-16.9)$ & .057 \\
\hline Sensor 2 (middle left side of cheek) & $10.5(6.2-13.8)$ & $7.7(6.7-11.8)$ & .17 \\
\hline Sensor 3 (lower left side of cheek) & $13.5(10.3-17.8)$ & $14.7(10.1-18.1)$ & .63 \\
\hline Sensor 4 (left side of chin) & $7.3(6.7-9.6)$ & $12.4(9.5-17.0)$ & .003 \\
\hline Sensor 5 (middle of chin) & $9.5(7.7-13.8)$ & $16.6(12.5-28.4)$ & $<.001$ \\
\hline Sensor 6 (right side of chin) & $13.5(10.9-14.9)$ & $16.9(15.1-25.6)$ & $<.001$ \\
\hline Sensor 7 (lower right side of cheek) & $10.0(8.5-11.3)$ & $10.0(9.0-11.3)$ & .77 \\
\hline Sensor 8 (middle right side of cheek) & $18.8(15.3-19.8)$ & $18.9(17.9-19.5)$ & .97 \\
\hline Sensor 9 (upper right side of cheek) & $5.6(2.6-6.7)$ & $5.4(3.3-7.0)$ & .55 \\
\hline Sensor 10 (left side of forehead) & $3.4(2.0-3.8)$ & $3.3(2.3-4.2)$ & .91 \\
\hline Sensor 11 (right side of forehead) & $13.2(3.1-13.5)$ & $13.0(3.1-13.7)$ & $>.99$ \\
\hline Sensor 12 (nasal bridge) & $32.3(23.0-46.2)$ & $18.6(15.6-24.4)$ & $<.001$ \\
\hline Distribution of contact pressure, $\%$ & $71.6(50.7-81.8)$ & $57.5(51.8-61.3)$ & .01 \\
\hline
\end{tabular}

$N=20$. Data are reported as median (interquartile range)

Mean values of contact pressure measured 3 times for $1 \mathrm{~min}$ in each sensor after applying the NIV mask with or without the fitting device and adjusting the leakage volume to $\sim 50$ L/min and distribution of contact pressure.

incidence because its use is not dependent on the clinician's skill.

In this study, the use of this fitting device resulted in blanchable erythema in 5 subjects even when the contact pressure was reduced. Use of a fitting device requires greater strap tension than a NIV mask only to maintain the fitting device on the face. The weight of the fitting device may have been the cause of the highcontact pressure around the chin as well as the shear stress on the nasal bridge, which is a known risk factor for NIV mask-related pressure ulcers. ${ }^{6}$ Therefore, we plan to use lighter materials to create the fitting devices in future studies.

This study has several limitations. First, the subjects were not blinded to intervention assignment, which might have affected the reporting of discomfort level. Second, our study was limited to Asian participants with a limited variety of facial characteristics. Third, the number of subjects was small and the subjects were healthy individuals, so the results may differ in patients who differ from the study population in terms of age, nutritional status, medication use, presence of comorbidities, and level of consciousness. ${ }^{13}$ Fourth, the short duration of the fitting device use did not allow an adequate assessment of the effects of the fitting device after 4-6 h under a sustained load, which would be an appropriate time frame for NIV mask-related pressure ulcers. ${ }^{34}$ Fifth, we could not consider other prevention strategies such as mask rotation because the duration of NIV mask application was limited.

Despite these limitations, we have proposed a promising method to prevent NIV mask-related pressure ulcers using 3D scanning solutions. The capacity of the proposed fit- ting device to prevent NIV mask-related pressure ulcers should be further investigated.

\section{Conclusion}

The use of the proposed personalized fitting device created with the aid of 3D scanning solutions reduced the incidence of blanchable erythema, standardized redness intensity, discomfort level, and contact pressure on the nasal bridge in healthy participants. The results of this study imply that the use of personalized fitting devices could prevent NIV mask-related pressure ulcers. Further studies are required to confirm the effectiveness of this device in the clinical setting.

\section{REFERENCES}

1. Pittman J, Beeson T, Kitterman J, Lancaster S, Shelly A. Medical device-related hospital-acquired pressure ulcers: development of an evidence-based position statement. J Wound Ostomy Continence Nurs 2015;42(2):151-154.

2. Murray JS, Noonan C, Quigley S, Curley MA. Medical devicerelated hospital-acquired pressure ulcers in children: an integrative review. J Pediatr Nurs 2013;28(6):585-595.

3. Apold J, Rydrych D. Preventing device-related pressure ulcers: using data to guide statewide change. J Nurs Care Qual 2012;27(1):28-34.

4. VanGilder C, Amlung S, Harrison P, Meyer S. Results of the 20082009 International Pressure Ulcer Prevalence Survey and a 3-year, acute care, unit-specific analysis. Ostomy Wound Manage 2009; 55(11):39-45

5. Coyer FM, Stotts NA, Blackman VS. A prospective window into medical device-related pressure ulcers in intensive care. Int Wound J 2014;11(6):656-664.

6. Fujimoto Y, Okuwa M, Nakatani T, Sanada H, Sato A. Risk factors of non-invasive positive pressure ventilation therapy mask-related pressure ulcers. J Tsuruma Health Sci Soc 2016;39(2):37-50. 


\section{Personalized Fitting Device to Prevent Pressure Ulcers}

7. Fauroux B, Lavis JF, Nicot F, Picard A, Boelle PY, Clement A, et al. Facial side effects during noninvasive positive pressure ventilation in children. Intensive Care Med 2005;31(7):965-969.

8. Rathore FA, Ahmad F, Zahoor MU. Case report of a pressure ulcer occurring over the nasal bridge due to a non-invasive ventilation facial mask. Cureus 2016;8(10):e813.

9. Lemyze M, Mallat J, Nigeon O, Barrailler S, Pepy F, Gasan G, et al. Rescue therapy by switching to total face mask after failure of face mask-delivered noninvasive ventilation in do-not-intubate patients in acute respiratory failure. Crit Care Med 2013;41(2):481-488.

10. Visscher MO, White CC, Jones JM, Cahill T, Jones DC, Pan BS. Face masks for noninvasive ventilation: fit, excess skin hydration, and pressure ulcers. Respir Care 2015;60(11):1536-1547.

11. Schallom M, Cracchiolo L, Falker A, Foster J, Hager J, Morehouse $\mathrm{T}$, et al. Pressure ulcer incidence in patients wearing nasal-oral versus full-face noninvasive ventilation masks. Am J Crit Care 2015; 24(4):349-356.

12. Kemalettin Y, Aykut Ö, Çetin D, Ethem G. An unusual location for a pressure ulcer: the nasal bridge. Wounds 2013;25(8):E9-E10.

13. Brill AK. How to avoid interface problems in acute noninvasive ventilation. Breathe 2014;10(3):230-242.

14. Weng MH. The effect of protective treatment in reducing pressure ulcers for non-invasive ventilation patients. Intensive Crit Care Nurs 2008;24(5):295-299.

15. Raurell-Torreda M, Argilaga-Molero E, Colomer-Plana M, Rodenas-Fransico A, Ruiz-Garcia MT, Uya Muntana J. Optimising noninvasive mechanical ventilation: which unit should care for these patients? A cohort study. Aust Crit Care 2017;30(4):225-233.

16. Schettino GP, Tucci MR, Sousa R, Valente Barbas CS, Passos Amato MB, Carvalho CR. Mask mechanics and leak dynamics during noninvasive pressure support ventilation: a bench study. Intensive Care Med 2001;27(12):1887-1891.

17. Dellweg D, Hochrainer D, Klauke M, Kerl J, Eiger G, Kohler D. Determinants of skin contact pressure formation during non-invasive ventilation. J Biomech 2010;43(4):652-657.

18. Nava S, Navalesi P, Gregoretti C. Interfaces and humidification for noninvasive mechanical ventilation. Respir Care 2009;54(1):71-84.

19. Yamaguti WP, Moderno EV, Yamashita SY, Gomes TG, Maida AL, Kondo CS, et al. Treatment-related risk factors for development of skin breakdown in subjects with acute respiratory failure undergoing noninvasive ventilation or CPAP. Respir Care 2014;59(10):15301536.

20. Da Silveira AC, Daw JL Jr., Kusnoto B, Evans C, Cohen M. Craniofacial applications of three-dimensional laser surface scanning. J Craniofac Surg 2003;14(4):449-456.

21. Rana M, Gellrich N-C, von See C, Weiskopf C, Gerressen M, Ghassemi A, et al. 3D evaluation of postoperative swelling in treatment of bilateral mandibular fractures using 2 different cooling therapy meth- ods: a randomized observer blind prospective study. J Cranio-Maxillofacial Surg 2013;41(1):e17-e23.

22. Amirav I, Luder AS, Halamish A, Raviv D, Kimmel R, Waisman D, et al. Design of aerosol face masks for children using computerized 3D face analysis. J Aerosol Med Pulm Drug Deliv 2014;27(4):272278.

23. Carroll A, Amirav I, Marchand R, Olmstead D, Logan H, Grosvenor A, et al. Three-dimensional modeled custom-made noninvasive positive pressure ventilation masks in an infant. Am J Respir Crit Care Med 2014;190(8):950.

24. Hsu D-Y, Cheng Y-L, Bien M-Y, Lee H-C. Development of a method for manufacturing customized nasal mask cushion for CPAP therapy. Australas Phys Eng Sci Med 2015;38(4):657-664.

25. Navalesi P, Fanfulla F, Frigerio P, Gregoretti C, Nava S. Physiologic evaluation of noninvasive mechanical ventilation delivered with three types of masks in patients with chronic hypercapnic respiratory failure. Crit Care Med 2000;28(6):1785-1790.

26. Girault C, Briel A, Benichou J, Hellot MF, Dachraoui F, Tamion F, et al. Interface strategy during noninvasive positive pressure ventilation for hypercapnic acute respiratory failure. Crit Care Med 2009; 37(1):124-131.

27. Black JM, Cuddigan JE, Walko MA, Didier LA, Lander MJ, Kelpe MR. Medical device related pressure ulcers in hospitalized patients. Int Wound J 2010;7(5):358-365.

28. Hanonu S, Karadag A. A prospective, descriptive study to determine the rate and characteristics of and risk factors for the development of medical device-related pressure ulcers in intensive care units. Ostomy Wound Manage 2016;62(2):12-22.

29. Makic MB. Medical device-related pressure ulcers and intensive care patients. J Perianesth Nurs 2015;30(4):336-337.

30. Yamamoto T, Takiwaki H, Arase S, Ohshima H. Derivation and clinical application of special imaging by means of digital cameras and Image $\mathbf{J}$ freeware for quantification of erythema and pigmentation. Skin Res Technol 2008;14(1):26-34.

31. Shikama M, Nakagami G, Noguchi H, Mori T, Sanada H. Development of personalized fitting device with three-dimensional solution for preventing medical device-related pressure ulcers caused by oralnasal mask during noninvasive positive pressure ventilation: a preliminary study (abstract). J Japan Soc Wound Ostomy Continence Manage 2016;20(2):185.

32. Dyer A. Ten top tips Preventing device-related pressure ulcers. Wounds In 2015;6(1):9-13.

33. Sugama J, Sanada H, Takahashi M. Reliability and validity of a multi-pad pressure evaluator for pressure ulcer management. J Tissue Viability 2002;12(4):148-153.

34. Bambi S, Peris A, Esquinas AM. Pressure ulcers caused by masks during noninvasive ventilation (letter). Am J Crit Care 2016;25(1):6. 